This item was submitted to Loughborough's Research Repository by the author.

Items in Figshare are protected by copyright, with all rights reserved, unless otherwise indicated.

\title{
Microneedle assisted transdermal delivery of zolmitriptan: effect of microneedle geometry, in vitro permeation experiments, scaling analyses and numerical simulations
}

PLEASE CITE THE PUBLISHED VERSION

http://dx.doi.org/10.1080/03639045.2017.1313862

PUBLISHER

(C) Taylor \& Francis

VERSION

AM (Accepted Manuscript)

\section{PUBLISHER STATEMENT}

This work is made available according to the conditions of the Creative Commons Attribution-NonCommercialNoDerivatives 4.0 International (CC BY-NC-ND 4.0) licence. Full details of this licence are available at: https://creativecommons.org/licenses/by-nc-nd/4.0/

\section{LICENCE}

CC BY-NC-ND 4.0

\section{REPOSITORY RECORD}

Uppuluri, Chandrateja, Jyothirmayee Devineni, Tao Han, Atul Nayak, Karthik J. Nair, Benjamin R. Whiteside, Diganta Das, and Buchi N. Nalluri. 2017. "Microneedle Assisted Transdermal Delivery of Zolmitriptan: Effect of Microneedle Geometry, in Vitro Permeation Experiments, Scaling Analyses and Numerical Simulations". figshare. https://hdl.handle.net/2134/24770. 


\section{MICRONEEDLE ASSISTED TRANSDERMAL DELIVERY OF ZOLMITRIPTAN: EFFECT OF MICRONEEDLE GEOMETRY, IN VITRO PERMEATION EXPERIMENTS, SCALING ANALYSES AND NUMERICAL SIMULATIONS}

Uppuluri, Chandra Teja; KVSR Siddahrtha College of Pharmaceutical Sciences Department of pharmaceutics

Devineni, Jyothirmayee; KVSR Siddahrtha College of Pharmaceutical Sciences, Department of pharmaceutics

Han, Tao; Loughborough University, Chemical Engineering Department

Nayak, Atul; Loughborough University, Chemical Engineering Department

Nair, Kartik J; University of Bradford, Department of Engineering and Informatics

Whiteside, Benjamin R.; University of Bradford, Department of Engineering and Informatics

Das, Diganta B.; Loughborough University, Chemical Engineering Department

Nalluri, Buchi N; KVSR Siddahrtha College of Pharmaceutical Sciences, Department of Pharmaceutics

\section{ABSTRACT}

Objective: The present study was aimed to investigate the effect of salient microneedle (MN) geometry parameters like length, density, shape and type on transdermal permeation enhancement of Zolmitriptan (ZMT).

Methods: Two types of MN devices viz. AdminPatch ${ }^{\circledR}$ arrays (ADM) (0.6, 0.9, 1.2 and $1.5 \mathrm{~mm}$ lengths) and laboratory fabricated polymeric MNs (PM) of $0.6 \mathrm{~mm}$ length were employed. In the case of PMs, arrays were applied thrice at different places within a $1.77 \mathrm{~cm}^{2}$ skin area (PM-3) to maintain the MN density closer to 0.6mm ADM. Scaling analyses was done using dimensionless parameters like concentration of ZMT $\left(\mathrm{C}_{\mathrm{t}} / \mathrm{C}_{\mathrm{s}}\right)$, thickness $(\mathrm{h} / \mathrm{L})$ and surface area of the skin $\left(\mathrm{Sa} / \mathrm{L}^{2}\right)$.

Results: Micro-injection moulding technique was employed to fabricate PM. Histological studies revealed that the PM, owing to their geometry/design, formed wider and deeper 
microconduits when compared to ADM of similar length. Approximately 3.17 and 3.65 fold increase in ZMT flux values were observed with 1.5mm ADM and PM-3 applications when compared to the passive studies. Good correlations were observed between different dimensionless parameters with scaling analyses. Numerical simulations, using MATLAB and COMSOL software, based on experimental data and histological images provided information regarding the ZMT skin distribution after MN application.

Discussion: Both from experimental studies and simulations, it was inferred that PM were more effective in enhancing the transdermal delivery of ZMT when compared to ADM.

Conclusion: The study suggests that MN application enhances the ZMT transdermal permeation and the geometrical parameters of MNs play an important role in the degree of such enhancement.

\section{INTRODUCTION}

Migraine is a chronic, debilitating headache affecting approximately $19 \%$ of the population globally, with 2-3 times more incidence in women than in men. It is a neurovascular disorder whose pathophysiology is still not clearly understood. An attack/episode of migraine may last from 2 to 72 hrs, associated with characteristic symptoms like a pulsating and throbbing headache generally on one side of the head, nausea, vomiting, sensitivity to light, etc that generally worsens with physical activity. Triptans are 5HT or Serotonin receptor agonists that act specifically on migraine. These cause constriction of the dilated cranial blood vessels, which is thought to be the cause of migraine ${ }^{1,2}$.

The first among this class of drugs is Sumatriptan, followed by newer ones viz. Zolmitriptan, Naratriptan, Rizatriptan, Eletriptan, Almotriptan, Avitriptan and Frovatriptan. Zolmitriptan (ZMT) was the second triptan available in the US and many countries, and was developed 
with the goal of creating a more bioavailable, longer half-life, more rapidly absorbed and centrally active oral tablet than Sumatriptan ${ }^{2}$. ZMT is available as oral tablet, nasal spray and oral dispersible tablet formulations, with an optimum dose being $2.5 \mathrm{mg}$ orally, with a maximum daily dose of $10 \mathrm{mg} / \mathrm{day}^{3}$. With all three formulations, the bioavailability is between 40-48\%. Nasal sprays of ZMT do offer a pharmacokinetic advantage of a rapid absorption across nasal mucosa, which results in a much faster onset of action. However, the overall therapeutic gain or efficacy is not improved significantly when compared to oral formulations. All these formulations of ZMT were reported to have a good chance of therapeutic failure with the first dose, requiring a second dose within $2 \mathrm{~h}$ to avoid a recurrence of the attack within $24 \mathrm{~h}^{3,4}$.

An ideal delivery system for ZMT must improve the bioavailability and ensure a good therapeutic gain within 2 h, i.e., a faster onset of action and a low 24 h recurrence rate when compared to existing options, without compromising patient compliance and ease of handling. Transdermal delivery has gained a lot of interest as an alternative to oral and parenteral routes, and research is going on extensively to open this route for systemic administration of a vast array of drug molecules including large molecules like insulin, growth hormone, etc ${ }^{5}$.

ZMT is a relatively hydrophilic molecule with a $\log \mathrm{P}$ value of 1.6 and does not have ideal properties to permeate through skin and hence a suitable permeation enhancement technique should be employed to achieve clinically significant rates of drug permeation. Only a few studies have reported on the transdermal delivery of ZMT, using chemical enhancers and iontophoresis as permeation enhancement techniques ${ }^{6-8}$. Subedi et al., $2011^{7}$ studied the effect of different formulation variables like the specific types of pressure sensitive adhesives (PSA), solvent system, permeation enhancers, crystallization inhibitors, etc. on transdermal 
flux of ZMT and reported that permeation enhancers (Polyoxyethylene alkyl ethers) resulted in a considerable increase in transdermal flux of ZMT, but induced ZMT crystallization ${ }^{7}$. Lui and Fang, $2015^{8}$, also developed an adhesive transdermal patch containing ZMT using DURO-TAK ${ }^{\circledR} 87-4098$ (PSA) and 10\% Azone as permeation enhancer at a drug load of 4\% ZMT and reported that $88 \pm 51 \mathrm{ng} / \mathrm{mL}$ ZMT was detected in systemic circulation within 15 min of administration of the patches in vivo using rabbit as an animal model, and concluded that further optimization can enable effective delivery of ZMT via a transdermal route ${ }^{8}$. Extensive work in the literature emphasizes that microneedles (MN), which are minimally invasive systems that can overcome the barrier functions of skin and improve the permeation of drug molecules including macromolecules, could be used to effectively enhance the permeation of ZMT via a transdermal route $\mathrm{e}^{9-14}$. It was also reported that such enhancement is dependent on many aspects of MN design, such as type, shape of MNs, density, materials, etc. There is also an interplay of the effect of these variables and, as such, their effects on the permeation of a drug molecule are generally non-intuitive. As a result, their effects are best studied for specific case $\mathrm{e}^{15,16}$.

Consistent with this idea, the present study was designed using a systematic approach to investigate the efficiency of MNs in enhancing the transdermal permeation of ZMT and to explore the effect of MN geometrical parameters viz. type, MN length, design/shape and density on the extent of ZMT transdermal permeation enhancement. Two types of MN devices viz. commercially available AdminPatch $^{\circledR}$ arrays (ADM) (0.6, 0.9, 1.2, $1.5 \mathrm{~mm}$ length) and lab fabricated polymeric MN arrays (PM) (0.6 mm length) of different $\mathrm{MN}$ densities were employed for the same purpose (Fig. 1). A number of different techniques such as etching, lithography and moulding were discussed widely in the literature for the manufacture of MNs of different dimensions (100-1800 $\mu \mathrm{m}$ in length), shapes with different materials (plastics, silicon, ceramics, metals, etc.). Injection moulding offers several 
advantages in terms of ease of scalability for bulk manufacture, range of materials and good reproducibility ${ }^{17}$. In this study, micro-injection moulding was employed for fabrication of the polymeric MN arrays (PM) using PEEK LT-3 (polyether ether ketone) as reported in Nair $\boldsymbol{e t}$ $a \mathbf{l}^{18}$.

\section{MATERIALS AND METHODS}

\section{Materials}

AdminPatch ${ }^{\circledR}$ MN arrays were purchased from AdminMed, Sunnyvale, U.S.A. Zolmitriptan was obtained from Mylan Laboratories Limited, Hyderabad, India; Sodium chloride, isopropyl alcohol, propylene glycol and 1\%w/v Safranin solution from Loba Chemie, Mumbai, India; Formic acid, acetonitrile, methanol and HPLC water from Merck Specialities Pvt. Ltd, Mumbai, India; Haematoxylin and Eosin stain from Sigma-Aldrich, Bengaluru, India. All the reagents and chemicals used in the study were of HPLC grade. Pig ear skin was obtained from local abattoirs.

\section{Fabrication of Polymeric MN (PM)}

MN array components consisted of 25 conical needles, each $0.6 \mathrm{~mm}$ in length with a base diameter of $0.3 \mathrm{~mm}$, in a $5 \times 5$ array over a $0.5 \times 0.5 \mathrm{~cm}^{2}$ area, supported by a circular substrate of diameter $17.5 \mathrm{~mm}$ and thickness $0.5 \mathrm{~mm}$. Moulding of these components was performed using a Wittmann-Battenfeld Micro-Power 15 micro-injection moulding machine as shown in Fig 2A. This machine offers a high repeatability, accurate dosing, clean room facility, and robotic component handling which is ideal for the bulk manufacture of MNs. The MN mould insert (Fig 2 B) was made out of Stavax ESR (Bohler-Uddeholm Corp, Elgin, USA). Sink electrical discharge machining (EDM) was used to create negative MN features into the insert and was outsourced to Isometric tool and design Inc. (New Richmond, USA). 
The polymer used for this study is PEEK (Polyetheretherketone) LT-3 grade material, which is an FDA-approved semi-crystalline biomaterial manufactured by Invibio Inc, Lancashire, UK and is widely employed for medical use. The attractive characteristics of PEEK LT-3 for this application include its excellent processability, dimensional stability, mechanical strength, chemical resistance and resistance to gamma and ethylene oxide sterilisation methods. To ensure the moisture content of the material was suitable for processing, the material was dried at a temperature of $150{ }^{\circ} \mathrm{C}$ for 4 hours prior to moulding.

Moulding trials were performed to optimise the process parameters based on measurement of the needle dimensions and substrate flatness. The optimised parameters for the moulding of PM are shown Table 1. Once the process was stabilised, samples were collected for the subsequent experimental work.

\section{MN dimensional evaluation}

Scanning electron microscope:

A Hitachi TM-3000 table-top Scanning Electron Microscope was used for analysis of the MN insert and needle geometry. The Hitachi TM-300 has a magnification from 15X to 30000X, sample chamber of $700 \mathrm{~mm}$ diameter and $50 \mathrm{~mm}$ thickness. It features $5 \mathrm{kV}, 15 \mathrm{kV}$ and analysis observation modes. Because of the high aspect ratio and low contrast of the MNs, analysis mode was used for imaging. The major advantage of using TM 3000 SEM was it works under low vacuum and does not need specimen coating.

Confocal laser microscope:

It is very important to measure the tip radius and height of the MNs and the most common methods used are optical or electron microscopy. Because of its steep and complex structures, a 3D image analysis gives a better measurement of the needle geometry and quality control. 
In this study an Olympus vertical scanning laser confocal microscope LEXT OLS 4000 was used to accurately measure the tip radius and height of MN arrays.

The device offers a broad magnification range from $108 \mathrm{X}$ to $17280 \mathrm{X}$ and the exact $3 \mathrm{D}$ reconstruction of the MNs. The confocal laser microscope LEXT scans the surfaces with a laser beam with the wavelength of $405 \mathrm{~nm}$ thus allowing submicron visualization of material and component surfaces with the resolution of down to $0.10 \mu \mathrm{m}$. Measurements were taken with the 20X lens using the wide range stitching feature with 20 percent overlap to produce a measurement area of $5 \mathrm{~mm} \times 5 \mathrm{~mm}$. A five level brightness switch (Table 2) was enabled to accurately illuminate the specimen.

Atomic force microscope:

The most important feature of the MN is that the tip has to be sharp in order to effectively pierce the skin. So as to confirm the measurements made from confocal laser microscope, AFM (MFP-3D Asylum Research Santa Barbara, USA) was used to measure the tip radius of the MNs. All AFM scans were made using an MFP-3D scanner from Asylum Research, Santa Barbara, USA. Silicon nitride cantilever tips (Applied Nanostructures, Santa Clara, USA) with a tip radius of $15 \mathrm{~nm}$ and spring constant of $0.3 \mathrm{Nm}$ were used. Images were interpreted using integrated MFP-3D ${ }^{\mathrm{TM}}$ Igor software (USA). Errors in the piezo-linearity were corrected for by using zero and first order flattening.

\section{HPLC method}

RP-HPLC-PDA method was developed specifically for the analysis of ZMT in the transdermal permeation samples. A Shimadzu Prominence HPLC system provided with DGU-20A3 degasser, LC-20AD binary pumps, SIL-20AHT auto sampler and SPD-M20A PDA detector was used for analysis of the samples. Data acquisition was carried out using 
LC solutions software. Separation was achieved on an Agilent Eclipse column $(150 \times 4.6$ $\mathrm{mm} ; 5 \mu \mathrm{m})$. The mobile phase comprised of $0.02 \% \mathrm{v} / \mathrm{v}$ formic acid : methanol 75:25 (v/v) at a flow rate of $1 \mathrm{~mL} / \mathrm{min}$ with an injection volume of $20 \mu \mathrm{L}$ and the eluents were monitored at $221 \mathrm{~nm}$. The developed method was validated as per ICH guidelines.

\section{Solubility studies}

The solubility of ZMT was studied in different vehicle combinations of propylene glycol (PG), polyethylene glycol-400 (PEG), and saline (S) at 70:30, 80:20 and 90:10\% v/v ratios. To each vehicle system, an excess amount of ZMT was added and vortexed for 1 min in order to obtain a saturated solution and the solutions were equilibrated at $37^{\circ} \mathrm{C}$ in an orbital shaker for $24 \mathrm{~h}$. After equilibration, the samples were centrifuged at $3000 \mathrm{rpm}$ for $10 \mathrm{~min}$ and filtered through a nylon syringe filter $(0.45 \mu \mathrm{m})$ and all the samples were appropriately diluted and analysed by HPLC method.

\section{Skin Preparation}

Pig ears were collected from the local abattoirs immediately after animals were killed by electric current. The ears were transported to the laboratory in a cooling box without previous treatment. In the laboratory, the pig ears were washed carefully with distilled water and the hair was removed from the external part of pig ear using an electrical hair clipper. Carefully the full-thickness skin from the external part of the pig ear was separated from underlying cartilage using a scalpel and excess fat underlying the skin was removed to a thickness of $1.2 \mathrm{~mm}$ was employed for the in vitro transdermal permeation studies. The dermis side was wiped with isopropyl alcohol to remove the residual adhering fat. Processed skin samples were individually wrapped in plastic bags without air entrapment and stored in a deep freezer at $-20^{\circ} \mathrm{C}$ until further use. 


\section{Application of MNs on skin samples}

Prior to the in vitro skin permeation experiments, the skin samples were allowed to be brought to room temperature and then the skin surface was carefully washed with saline. Two types of MN devices viz, $\mathrm{ADM}(0.6,0.9,1.2$ and $1.5 \mathrm{~mm})$ and $\mathrm{PM}(0.6 \mathrm{~mm})$ were used to poke the skin surface under thumb pressure. In the case of PM, both single (PM-1) and triple (PM-3) insertions at different places within a $1.77 \mathrm{~cm}^{2}$ skin area (PM-3) were made in order to maintain the MN density closer to ADM of $0.6 \mathrm{~mm}$ length as shown in Fig 1 . The MNs were periodically checked during this process for potential damage using a stereomicroscope.

\section{Surface visualization and histological examination of the skin samples}

To visually confirm the disruption of skin layers by the MNs, the arrays were pressed over the pig ear skin under thumb pressure and held for $1 \mathrm{~min}$. Then the skin was stained with safranin dye $(1 \% \mathrm{w} / \mathrm{v}$ in water) and wiped with isopropanol cotton swabs for the identification of the microconduits formed. In the case of the histological studies, the skin section samples with and without $\mathrm{MN}$ treatments were prepared after staining with haematoxylin and eosin for visualization of skin layers and to display a clear indentation by MN penetration. The sections were observed under a microscope (Olympus; Noida, India). The width and depth of microconduits formed were also calculated in triplicate ( $\mathrm{n}=3$ skin samples for each MN) with the help of Toup View 3.2 Software (Irwin, U.S.A). For control, skin samples without MN treatment were also prepared.

\section{In Vitro Skin Permeation Studies}

The in vitro transdermal permeation studies were performed using a vertical type Franz diffusion cell apparatus fitted with a water circulation system, a water heater and an eight 
stage magnetic stirrer (Orchid Scientifics, Nasik, India). Franz diffusion cells with an effective diffusion area of $1.77 \mathrm{~cm}^{2}$ and a receptor volume of around $14 \mathrm{~mL}$ were used. Saline was used as the receptor fluid. Pig ear skin was mounted between the donor and receptor cells with the SC facing towards the donor cell. The receptor medium was stirred for uniform drug distribution at a speed of $600 \mathrm{rpm}$ throughout the experiment. Care was taken to prevent the entrapment of air bubbles at the interface of the skin (dermis) and the receptor solution. The surface of the skin was maintained at $32^{\circ} \mathrm{C}$ using a circulating water bath. After equilibration, $500 \mu \mathrm{L}$ of donor solutions containing excess amount of ZMT were applied on to the skin. Samples $(500 \mu \mathrm{L})$ were withdrawn from the receptor fluid at six hour increments up to 48 hours and replaced with the fresh saline to maintain a constant volume. All the samples were stored at $4^{\circ} \mathrm{C}$, prior to the HPLC analysis.

The cumulative permeation profiles were plotted for the cumulative amount of drug permeated $\left(\mathrm{nmoL} / \mathrm{cm}^{2}\right)$ as a function of time, for untreated and microneedle treated skin. The flux values and the respective lag times were obtained from the slope and the $\mathrm{X}$-intercept of the steady state portion of the cumulative permeation profiles. Apparent permeability and diffusion coefficient values were computed from Fick’s first law of diffusion:

$$
\frac{1}{A}\left(\frac{d M}{d t}\right)=J_{s}=K_{p} \Delta C
$$

$\boldsymbol{J}_{\boldsymbol{s}}$ is the steady-state flux $\left(\mathrm{nmoL} / \mathrm{cm}^{2} / \mathrm{hr}\right), \boldsymbol{M}$ is the cumulative amount of drug permeating the skin $\left(\mathrm{nmoL} / \mathrm{cm}^{2}\right), \boldsymbol{A}$ is the area of the skin $\left(1.77 \mathrm{~cm}^{2}\right), \boldsymbol{K}_{\boldsymbol{p}}$ is the apparent permeability coefficient $(\mathrm{cm} / \mathrm{hr})$, and $\Delta \boldsymbol{C}$ is the difference in concentrations of ZMT in the donor and receiver. Sink conditions were maintained in the receiver throughout the experiment and hence $\boldsymbol{\Delta C}$ was approximated to be the drug concentration in the donor compartment. 
Enhancement ratios were also computed to evaluate the relative efficiency of different MNs on the ZMT skin permeation enhancement. The enhancement ratios were calculated as follows:

$$
\text { Enhancement Ratio }=\frac{\text { Cumulative amount or Flux obtained after MN application }}{\text { Cumulative amount or Flux obtained from passive studies }}
$$

\section{ZMT content in skin}

After the completion of the permeation studies, skin samples were studied for drug disposition. The skin tissue exposed to the donor solution was cut with a scalpel and washed with filtered water and blotted with a paper towel in order to remove the drug which adhered to the surface. Then skin was minced with a scalpel, and placed in a pre-weighed vial. The drug was extracted from the skin by equilibrating with $5 \mathrm{~mL}$ of acetonitrile at $32^{\circ} \mathrm{C}$ in an orbital shaker. The solutions were then analyzed by HPLC to determine the ZMT content.

\section{Scaling analyses}

Dimensionless correlations between the amount of ZMT permeated $\left(\mathrm{C}_{t} / \mathrm{C}_{\mathrm{s}}\right)$ and other variables such as the thickness $(h / L)$ and the surface area $\left(S a / L^{2}\right)$ of the skin were made in order to gain insights into the overall phenomenon of ZMT transdermal permeation enhancement by MN application. The scaling analyses were carried out based on the principles of the Buckingham $\pi$ theorem where it is defined that the dimensionless concentration of a drug, which permeates through skin, can be defined in terms of key nondimensional parameters (e.g., MN lengths) using the procedures described in previous papers $^{13,19}$. 
Eq.1 describes the relationship of all the model parameters used for such analyses (Table 3).

$$
\frac{C_{t}}{C_{s}}=K\left[\frac{S_{a} L^{4} K_{e}}{V_{d} h D}\right]^{n}
$$

Where, ' $K$ ' is a dimensionless constant and ' $n$ ' is an unknown power; $C_{t}$ and $C_{s}$ are the amount of ZMT permeated at a given time t (48 h) and the amount of drug loaded in the donor compartment for diffusion (surface concentration on skin); $S_{a}$ is the surface area of skin available for diffusion; $L$ is the length of microneedles; $K_{e}$ and $V_{d}$ are the first order elimination constant and the volume of receptor fluid; ' $h$ ' is the thickness of skin and D is the diffusion coefficient of ZMT in skin.

Using Eq.1, the correlations between the dimensionless ZMT concentration $\left(\mathrm{C}_{t} / \mathrm{C}_{\mathrm{s}}\right)$ against the dimensionless parameters of the study $\left(\mathrm{h} / \mathrm{L}\right.$ and $\mathrm{S}_{\mathrm{a}} / \mathrm{L}^{2}$ ) have been established considering that all other variables remain unchanged.

\section{Numerical simulations of experimental studies}

A set of well-defined numerical simulations were carried out in order to gain insights into the specific effects of $\mathrm{MN}$ shape, dimensions, force of insertion of $\mathrm{MN}$, etc. on the overall permeation enhancement of ZMT, and also to obtain the information about ZMT distribution within the skin layers during permeation, which are otherwise difficult to obtain directly from the experimental data. The simulations were carried out using MATLAB (Math Works, MA, USA) and COMSOL Multiphysics (COMSOL Multiphysics Pvt. Ltd., Stockholm, Sweden) programmes. The MATLAB programme was use to process the histological sections images of skin treated with MNs and these processed images were further coupled with the experimental permeation parameter, e.g., passive diffusion coefficient and imported into COMSOL simulator software ${ }^{13,20}$. 


\section{Statistical analysis of the data}

Results of the experimental data were subjected to statistical analysis by one way ANOVA (using Fischer's LSD post hoc test) using SYSTAT 13 software (Systat Software Inc., San Jose, USA). Results with a $p$ value of less than 0.05 were considered to have statistically significant variance. Mean of replicate measurements $(n=3)$ with corresponding standard deviation (SD) was used to represent the data and to plot the graphs. 


\section{RESULTS}

\section{Fabrication and characterization of PM}

The actual dimensions of the PM were determined using 3D Laser Scanning Confocal Microscopy (LSCM) (Fig 3 A), atomic force microscopy (AFM) (Fig. 3 B) and scanning electron microscopy (SEM) (Fig 3 C). The measurement data suggests an average microneedle form which was $556 \pm 30 \mu \mathrm{m}$ in height with a tip radius of $32 \pm 8 \mu \mathrm{m}$, a base diameter of $300 \mu \mathrm{m}$ and $\mathrm{MN}$ interspacing of approximately $1 \mathrm{~mm}$ at the base.

For better comparison and understanding of the difference in the shape/design between PM and $\mathrm{ADM}$ devices, 3D confocal images of the $0.6 \mathrm{~mm} \mathrm{ADM}$ were recorded and are shown in Fig 3 D. Also the difference in base width among the devices can be clearly observed from the Fig 1. The various geometry parameters of both types of MN devices are given in Table 4.

\section{Analytical Method}

A rapid and sensitive HPLC-PDA method was developed for the selective quantification of ZMT in transdermal permeation studies. Under the conditions, ZMT eluted at 2.8 min (Fig 4 A) with good peak shape. Also, the specificity of the method to ZMT was demonstrated by the UV spectrum and the peak purity index curves (Fig 4 B, C). The method was validated as per ICH guidelines and complied with all the requirements.

\section{Solubility Studies}

Solubility studies for ZMT were performed with a view to select an appropriate donor vehicle for conducting in vitro skin permeation studies. PG:S and PEG:S combinations at 70:30, 
80:20 and 90:10 \%v/v were studied for ZMT solubility. Solubility data of ZMT is shown in Fig 5.

\section{Surface visualization and histological examination of skin samples}

In order to visually confirm the disruption of skin layers by $\mathrm{MN}$ insertion, the arrays were pressed over the pig ear skin under thumb pressure and held for 1 min. Following removal of the arrays, the skin samples were stained with safranin $(1 \% \mathrm{w} / \mathrm{v})$. The digital photographs of MN treated skin are shown in Fig 6.

Histological images of skin treated with MNs are shown in Fig 7. From the images, the stratum corneum disruption and the formation of microconduits across skin layers was clearly evident. The average penetration depth (vertical) $(n=3)$ of the MNs was found to be 180.26 \pm 30.39, $302.22 \pm 22.28,400.85 \pm 20.83,478.48 \pm 67.72$ and $338.20 \pm 22.66 \mu \mathrm{m}$ for ADM (0.6, 0.9, 1.2, $1.5 \mathrm{~mm})$ and PM, respectively.

\section{In vitro permeation studies}

The comparative in vitro pig ear skin permeation profiles of ZMT without and with MN application were shown in Fig 8. Various ZMT permeation parameters viz. cumulative amount permeated at the end of $48 \mathrm{~h}$, steady state flux, lag time, permeability and diffusion coefficients without and with microneedle treatment were given in Table 5.

\section{Scaling analyses}

Using scaling analyses, correlations were developed between the dimensionless parameters, namely, the amount of ZMT permeated $\left(\mathrm{C}_{\mathrm{t}} / \mathrm{C}_{\mathrm{s}}\right)$ and skin thickness $(\mathrm{h} / \mathrm{L})$ and surface area of skin $\left(\mathrm{S}_{\mathrm{a}} / \mathrm{L}^{2}\right)$ in order to derive a better understanding on the effect of specific geometry 
parameters of $\mathrm{MN}$, like length (L), on overall permeation enhancement by MNs of same design (ADM) (Fig 9A, B ,respectively) $)^{13,19}$. Eqs. 2 and 3 describe such relationships among the considered parameters within the given range. These correlations were determined for given thickness and surface area of skin while the MN length varies (0.6, 0.9, 1.2 and 1.5 $\mathrm{mm})$.

$$
\begin{gathered}
\frac{C_{t}}{C_{s}}=5.52 \times 10^{-3}\left[\frac{h}{L}\right]^{-0.624} \quad \text { for } \quad 0.8 \leq \frac{h}{L} \leq 2 \text {---- (2) } \\
\frac{C_{t}}{C_{s}}=2.47 \times 10^{-2}\left[\frac{S_{a}}{L^{2}}\right]^{-0.312} \quad \text { for } \quad 78.5 \leq \frac{S_{a}}{L^{2}} \leq 491.5 \text {---- (3) }
\end{gathered}
$$

Good correlation was observed $\left(\mathrm{R}^{2}>0.83\right)$ between the dimensionless parameters of the study (Fig 9).

\section{Numerical simulations of experimental studies}

The numerical simulations were carried out using the procedures discussed in previous papers based on in vitro passive diffusion coefficient values, coupled with histological section images (of corresponding MNs) ${ }^{13,20}$. Different stages in processing the histological section images by MATLAB and COMSOL programs for simulations are shown in Fig 10. The numerical simulations were able to provide information regarding the skin distribution of ZMT after applying the MNs at any time point and depth (Fig 10D).

\section{DISCUSSION}

The dimensions of the PM were found to be consistent and repeatable with good tip shape, confirming the complete filling of the PEEK into the MN insert cavity under the maintained processing conditions (Table 1) and the technique used is reliable for the bulk manufacture of PMs. Compression tests of PM on a steel plate using Bose Electroforce 3100 instrument with 
a $225 \mathrm{~N}$ load cell and Wintest ${ }^{\circledR}$ software (Bose, MN, USA) revealed that the PMs were able to withstand compression forces of up to $8 \mathrm{~N}^{18}$.

A rapid and sensitive HPLC-PDA method was developed for the selective quantification of ZMT in transdermal permeation studies. The method complied with all the requirements of ICH guidelines and was successfully employed for the quantitative estimation of ZMT in various samples throughout the study.

Solubility of ZMT was relatively higher in PG:S when compared with PEG:S combinations ( $p>0.05$ ) (Fig 5). However, a significant difference was not observed among PG:S vehicle combinations $(p>0.05)$. PG:S at $70: 30 \% \mathrm{v} / \mathrm{v}$ was selected for the ZMT in vitro skin permeation studies.

Surface visualization of MN treated skin showed clear distinctions in the number of the microconduits formed due to the differences in length and density of MNs in the ADM and PM devices (Fig 6). Skin layer disruption and the formation of microconduits was clearly evident from histological section images (Fig 7). The penetration depth about $25-35 \%$ of the original needle length for ADM and 55-60\% for the PM MN. Even though the length of the MNs differs in ADM, the percentage of MN penetration is almost same, which is an indication of uniformity in thumb pressure under which MNs were applied at different times.

With the ADM devices, as the length of the MNs increased the penetration depth also increased. However, the microconduits were found to be wider and deeper with PM when compared to ADM of similar lengths i.e. $0.6 \mathrm{~mm}$. These differences in the efficiency of creating microconduits in skin layers between the two types of MN devices (ADM and PM) may be attributed to the differences in geometry parameters like shape, design and type of 
fabricating material. Regarding the shape/design, the PMs were conical (3D) in shape, and the microconduits formed by PM were wider, while the microconduits formed using ADM looked merely like a cut on the skin (2D) as the arrays of ADM are low in thickness (2D) (Fig 3). Furthermore, owing to the sturdy and thick base (when compared to ADM) that supports the solid PM (Fig 1), the effective resistance (because of the viscoelastic nature) of the skin during application of arrays may be less for PM, while the ADM might have experienced greater resistance which is because of their thin base and array design ${ }^{21}$, resulting in a relatively shallow penetration with similar MN lengths (0.6 and 0.9 mm ADM).

Significant enhancement in ZMT permeation was observed after the application of MNs into the skin $(p<0.05)$ when compared to passive permeation studies (Fig 8). A 1.83, 1.91, 2.35 and 3.17 fold increase in the cumulative amount (48 h) of ZMT permeated was observed with 0.6, $0.9,1.2$ and $1.5 \mathrm{~mm}$ ADM treatments, respectively, when compared to the passive permeation amount. A similar trend was observed with other permeation parameters like permeability and diffusion coefficient values (Table 6). Significant reduction in the lag time was observed after the application of the $1.5 \mathrm{~mm}$ ADM when compared to that with the passive data and other ADM lengths $(p<0.05)$. However, there is no statistically significant difference between the 0.9 and $1.2 \mathrm{~mm}$ ADM treatments in terms of lag time $(p>0.05)$.

The ZMT flux values were found to be in the order of $1.5 \mathrm{~mm}>1.2 \mathrm{~mm}>0.9 \mathrm{~mm} \geq 0.6 \mathrm{~mm}>$ passive with ADM treatments. A 2.26 fold increase in ZMT flux was observed with the 1.5 mm when compared to passive studies. However, there is no statistically significant difference between the 0.6 and $0.9 \mathrm{~mm}$ treatments $(p>0.05)$. It was observed that the enhancement of ZMT permeation was only marginal with the application of the 0.6 and 0.9 mm ADM when compared to passive studies (Fig 8). This may be due to the fact that the skin 
disruption/penetration caused by 0.6 and $0.9 \mathrm{~mm}$ ADM was less, as supported by the histological section images (Fig 7). Moreover, the ZMT permeation was high with the 1.5 mm ADM when compared to $0.6,0.9$ and $1.2 \mathrm{~mm}$ even though the density of the MNs is low, which is possibly due to longer needle lengths which, in turn, resulted in deeper skin penetration.

With the PM application, approximately 1.37 and 3.65 fold increase in the cumulative amount (48 h) of ZMT permeated was observed with the PM-1 and PM-3 treatments, respectively, when compared to passive permeation amounts. The lag times were found to be significantly lower for PM-3 when compared to passive and PM-1 treatments $(p<0.05)$. The ZMT flux values were found to be in the order of PM-3 > PM-1 > passive treatments. A 1.25 and 2.96 fold increase in ZMT flux was observed with the PM-1 and PM-3, respectively, when compared to passive studies. A similar trend was observed with other ZMT permeation parameters like permeability and diffusion coefficient values (Table 5) with both the PM treatments.

Even though, the skin penetration by PM device (with $0.6 \mathrm{~mm}$ array) was significantly greater when compared to 0.6 and $0.9 \mathrm{~mm} \mathrm{ADM} \mathrm{(Fig} \mathrm{7),} \mathrm{the} \mathrm{overall} \mathrm{permeation} \mathrm{enhancement} \mathrm{in}$ terms of flux, cumulative amount permeated etc. achieved with PM-1 was found to be closer to those of 0.6 and $0.9 \mathrm{~mm}$ ADM ( $p>0.05$ ). This may be because the needle density of 0.6 and $0.9 \mathrm{~mm}$ ADM was greater when compared to PM-1 (Table 4), which may have compensated the variation in the extent of skin penetration among these MNs, and thus no significant variation in overall permeation enhancement was observed among these three MNs $(p>0.05)$. 
In the case of the PM, a single application of array didn't cover the $1.77 \mathrm{~cm}^{2}$ surface area of the skin and hence in order to maintain the MN density closer to the ADM of $0.6 \mathrm{~mm}$ length, the arrays were applied three times at different places within a $1.77 \mathrm{~cm}^{2}$ skin area (PM-3) as shown in Fig 1. As the needle density was increased with PM-3, the ZMT transdermal permeation increased markedly and was similar to that obtained with $1.5 \mathrm{~mm}$ ADM ( $p>0.05)$.

Moreover, it is intriguing to note although the needle density of PM-3 (75 MNs) was significantly greater when compared to the $1.5 \mathrm{~mm}$ ADM (31 MNs), the lag times were found to be significantly shorter with $1.5 \mathrm{~mm}$ ADM $(p<0.05)$, which may be because of deeper (vertical) penetration into skin. Even though the depth of the penetration was significantly lower with PM when compared to $1.5 \mathrm{~mm}$ ADM, the comparable permeation enhancement by PM-3 is may be because of higher needle density as stated earlier and wider microconduits formed as evidenced by histological section images (Fig 7).

The enhancement in ZMT permeation was found to be in the order of $1.5 \mathrm{~mm}$ ADM $\geq \mathrm{PM}-3>$ $1.2 \mathrm{~mm} \mathrm{ADM}>0.9 \mathrm{~mm}$ ADM $\geq 0.6 \mathrm{~mm}$ ADM $\geq \mathrm{PM}-1>$ Passive. Even though no correlation of ZMT skin content was observed with different MN treatments, significantly higher amounts of ZMT were found to be distributed in skin layers at the end of $48 \mathrm{~h}$ with $\mathrm{MN}$ treated studies and is an indication of potential ZMT skin deposition.

Patel et al., $2009^{6}$, investigated the effect of iontophoresis on transdermal permeation of ZMT both in vitro and in vivo. The cumulative amount of ZMT permeated was found to be approximately $263.7 \pm 92.7 \mu \mathrm{g} / \mathrm{cm}^{2}$, in vitro, at the end of $6 \mathrm{~h}$ with continuous application of current $\left(0.25 \mathrm{~mA} / \mathrm{cm}^{2}\right)$. However, the preliminary studies carried out in the present investigation showed a similar result (cumulative amount permeated) at the end of $48 \mathrm{~h}$ with 
1.5mm ADM and PM-3 applications $\left(251.08 \pm 17.07\right.$ and $289.85 \pm 64.58 \mu \mathrm{g} / \mathrm{cm}^{2}$ respectively).

Moreover, iontophoresis was reported to be associated with itching, redness and skin irritation and tingling sensation, making iontophoresis less patient compliant ${ }^{22}$. The MN application, especially the poke and patch technique discussed in this investigation, is painless and easy to administer when compared to iontophoresis, thus alleviating any issues regarding patient compliance.

Overall, further enhancement in the cumulative amount permeated and other permeation parameters like steady state flux of ZMT may be achieved by further optimizing the MN geometry, especially of the polymeric MNs (PM) in terms of design, MN length and density, and other parameters of the study like drug loading, donor vehicle, formulation, etc. thus resulting in more rapid and effective ZMT delivery.

Dimensionless correlations were developed for ADM MNs (same type but differing in length) using scaling analyses. Good correlations were observed between the dimensionless parameters $\left(C_{t} / C_{s} v s h / L\right.$ and $C_{t} / C_{s}$ vs $S_{a} / L^{2}$ ) (Fig 9). These correlations can be used to predict the amount of ZMT permeated with high accuracy for other MN lengths in the range of 0.6 1.5mm with similar design as ADM.

From the results of numerical simulations, the flux was represented by white arrows in Fig 10D showing obvious increment at the upper layers of the skin which is caused by MN penetration. This increment may be extrapolated to a higher drug accumulation rate at the blood stream as well as shorter lag time for the diffusion to reach its steady state after the application of MNs. Based on the simulation results, the diffusion profile of ZMT showed significant permeation increment with $1.5 \mathrm{~mm}$ ADM and PM when compared to other MNs 
in the study. Furthermore, the advantage of greater penetration by PM can be further magnified by increasing the MN density, as in the case of PM-3.

These simulations can be of great research value as they may be used to predict the degree of transdermal permeation enhancement of ZMT with other (new) dimensions and designs of MNs using the respective histological section images and the passive diffusion coefficient data.

\section{CONCLUSION}

Two types of MN devices (ADM and PM) were employed in this investigation to derive a greater understanding on the effects of various geometry parameters specifically on the transdermal permeation of ZMT by MN application. It was observed that the application of MNs significantly enhances the transdermal permeation of ZMT across pig ear skin. In

addition, the type/shape, density and more importantly, the length of MNs (depth of penetration into skin) were found to play a crucial role in the overall permeation enhancement of ZMT using this technique. It may be inferred that the transdermal delivery of ZMT, at clinically significant levels and in a painless and non-invasive manner, may by possible using MN applications. This investigation can form a basis for further studies (in vivo) and for optimization of various MN parameters to achieve successful delivery of ZMT via. MN assisted transdermal delivery systems.

\section{ACKNOWLEDGEMENTS}

The authors are thankful to Mylan Pharmaceuticals India Ltd, Hyderabad, for providing a gift sample of ZMT, to Dr. Naveen, Department of Pathology, Dr. Pinnamaneni Siddhartha Institute of Medical Sciences and Research Foundation, Vijayawada, for providing the required facilities for taking histological sections of skin samples and also to the Siddhartha 
Academy of General and Technical Education, Vijayawada, for providing necessary facilities to carry out the research work. The authors also extend their sincere thanks to DST, Ministry of Science and Technology, Govt. of India and the British Council, London, UK, for funding this research work under the DST-UKIERI scheme (DST/INT/UK/P-60/2014).

\section{DECLARATION OF INTEREST}

The authors declare no conflict of interest.

\section{REFERENCES}

1. Silberstein SD_Migraine symptoms: results of a survey of self-reported migraineurs. Headache. 1995; 35(7): 387-96.

2. Rapoport AM, Tepper SJ, Sheftell FD, Kung E, Bigal ME. Which triptan for which patient? Neurol. Sci. 2006; 27: S123-S129.

3. Mollie MJ, Alan MR. Triptans for the management of migraine. Drugs. 2010. 70: 1505-1616.

4. Marcelo EB, Carlos AB, Ana LA, José G. The triptan formulations - a critical evaluation. Speciali Arq Neuropsiquiatr. 2003; 61(2-A): 313-320.

5. Scheindlin S. Transdermal drug delivery: past, present, future. Mol Interv. 2004; 4(6): 308-312.

6. Patel SR, Zhong H, Sharma A, Kalia YN. Controlled non-invasive transdermal iontophoretic delivery of zolmitriptan hydrochloride in vitro and in vivo. Eur J Pharm Biopharm. 2009; 72: 304-309.

7. Subedi RK, Ryoo JP, Cheol M, Choi HK. Influence of formulation variables in transdermal drug delivery system containing Zolmitriptan. International journal of pharmaceutics. 2011; 419(1-2): 209-214. 
8. Lui C, Fang L. Drug in adhesive patch of Zolmitriptan: formulation and in virto/in vivo correlation. AAPS PharmaSciTech. 2015; 16(6): 1245-1253.

9. Ritesh K, Anil P. Modified transdermal technologies: breaking the barriers of drug permeation via the skin. Tropical Journal of Pharmaceutical Research. 2007; 6 (1): 633-644.

10. Jeong W, Lee A, Jung-Hwan PB, Prausnitz MR. Dissolving microneedles for transdermal drug delivery Biomaterials. 2008; 29: 2113-2124.

11. Zhou CP, Liu YL, Wang HL, Zhang PX, Zhang JL. Transdermal delivery of insulin using microneedle rollers in vivo. International Journal of Pharmaceutics. 2010; 39(2): 127-133.

12. Nalluri $\mathrm{BN}_{2}$ Sai Sri Anusha $\mathrm{V}_{2}$ Bramhini SR, Amulya J, Sultana AS,_Teja UC, Das DB. In vitro skin permeation enhancement of sumatriptan by microneedle application. Curr Drug Deliv. 2015; 12(6): 761-769.

13. Leeladurga V, Teja UC, Ashraf SS, Sundeep K, Sai Sri Anusha V, Han T, Nalluri BN, Das DB. Application of microneedle arrays for enhancement of transdermal permeation of Insulin: in vitro experiments, scaling analyses and numerical simulations. AAPS Pharma Sci Tech. 2015; 1-8. (DOI: 10.1208/S12249-015-0416-8).

14. Cheung K, Das DB. Microneedles for drug delivery: trends and progress. Drug Deliv. 2015. DOI:10.3109/10717544.2014.986309 (in press).

15. Chueng K, Han T, Das DB. Effect of force of microneedle insertion on the permeability of insulin in skin. J. Diabetes Sci. Technol. 2014; 8: 444-452.

16. Gomaa YA, Morrow DI, Garland MJ, Donnelly RF, El-Khordagui LK, Meidan VM. Effects of microneedle length, density, insertion time and multiple applications on human skin barrier function: assessments by transepidermal water loss. Toxicol. In Vitro. 2010; 24(7): 1971-1978. 
17. Attia UM, Marsona S, Alcock JR. Micro-injection moulding of polymer microfluidic devices. Microfluidics and nano fluidics. 2009; 7: 1-28.

18. Nair KJ, Whiteside BR, Grant C, Patel R, Tuinea-Bobe C, Norris K, Paradkar AR. Investigation of Plasma Treatment on Micro-Injection Moulded Microneedle for Drug Delivery, Pharmaceutics. 2015; 7: 471-485.

19. Al-Qallaf B, Das DB, Mori D, Cui Z. Modelling transdermal delivery of high molecular weight drugs from microneedle systems. Phil. Trans. R. Soc. A. 2007; 365: 2951-67.

20. Han T, Das DB. A new paradigm for numerical simulation of microneedle-based drug delivery aided by histology of microneedle-pierced skin. J. Pharm. Sci. 2015; 104(6): 1993-2007.

21. Vadim VY. 2010, US Patent No. 7658728 B2. Washington DC: U.S. Patent and Trademark Office.

22. Li GL, Van Steeg TJ, Putter H, Van Der Spek J, Pavel S, Danhof M, Bouwstra JA. Cutaneous side-effects of transdermal iontophoresis with and without surfactant pretreatment: a single-blinded, randomized controlled trial. Br. J. Dermatol. 2005; 153(2): 404-412. 

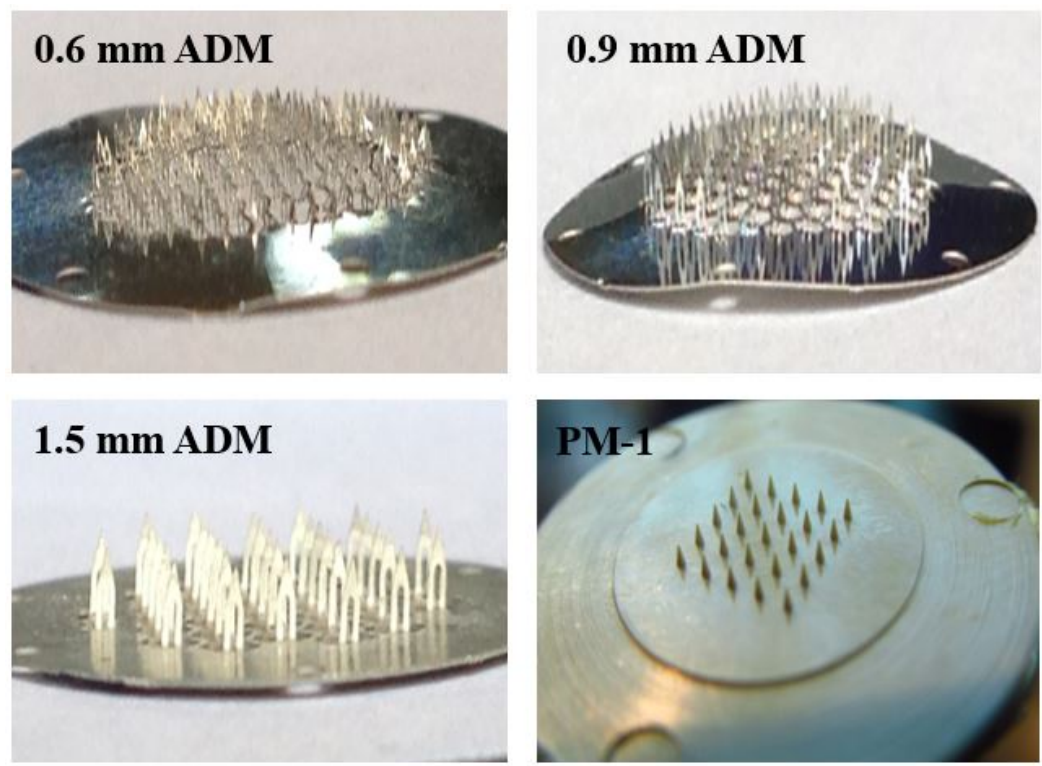
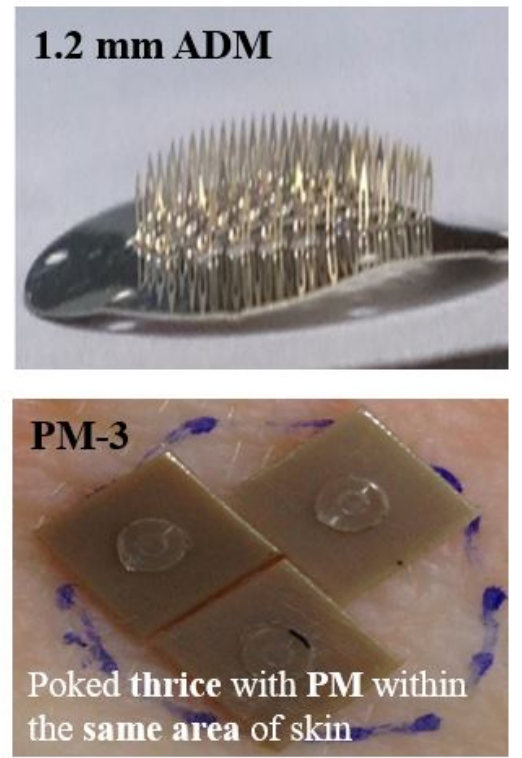

Fig 1. Different MNs used in the study 
$\mathbf{A}$

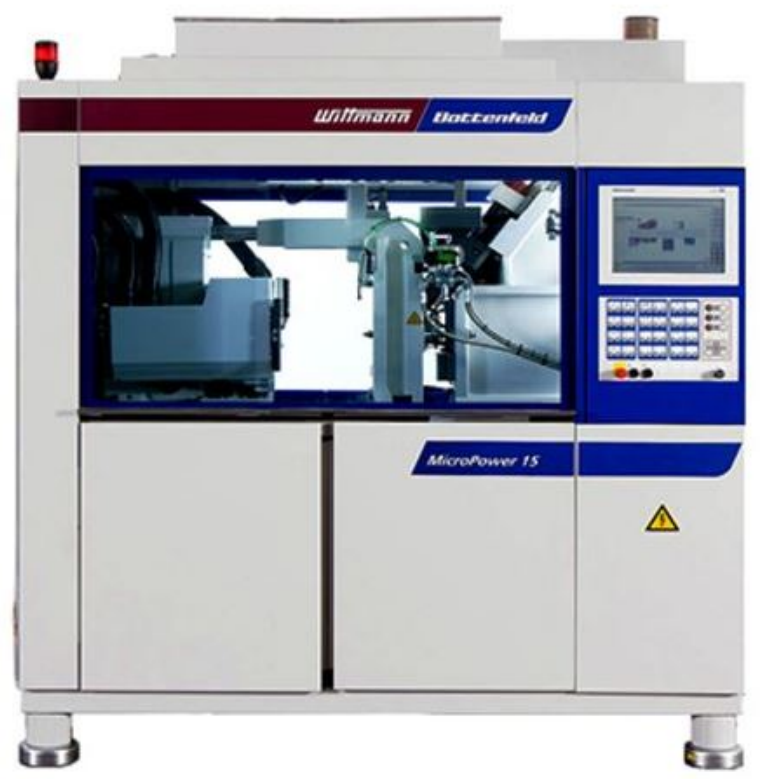

B

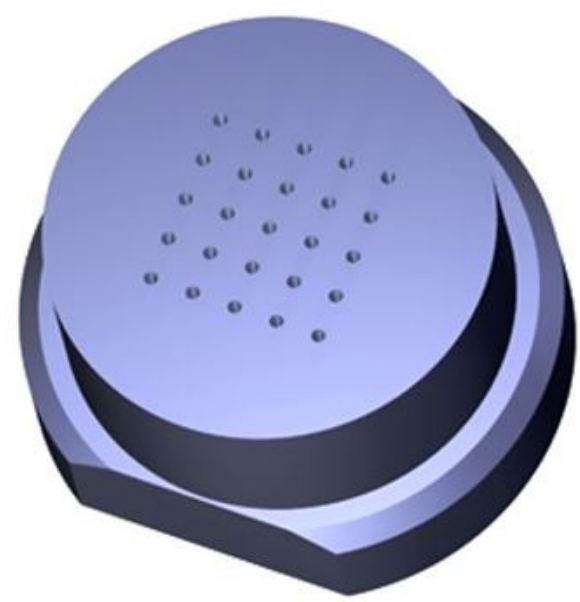

Fig 2. (A) Battenfeld micro-power 15 moulding machine; (B) 3D drawing of the microneedle insert 


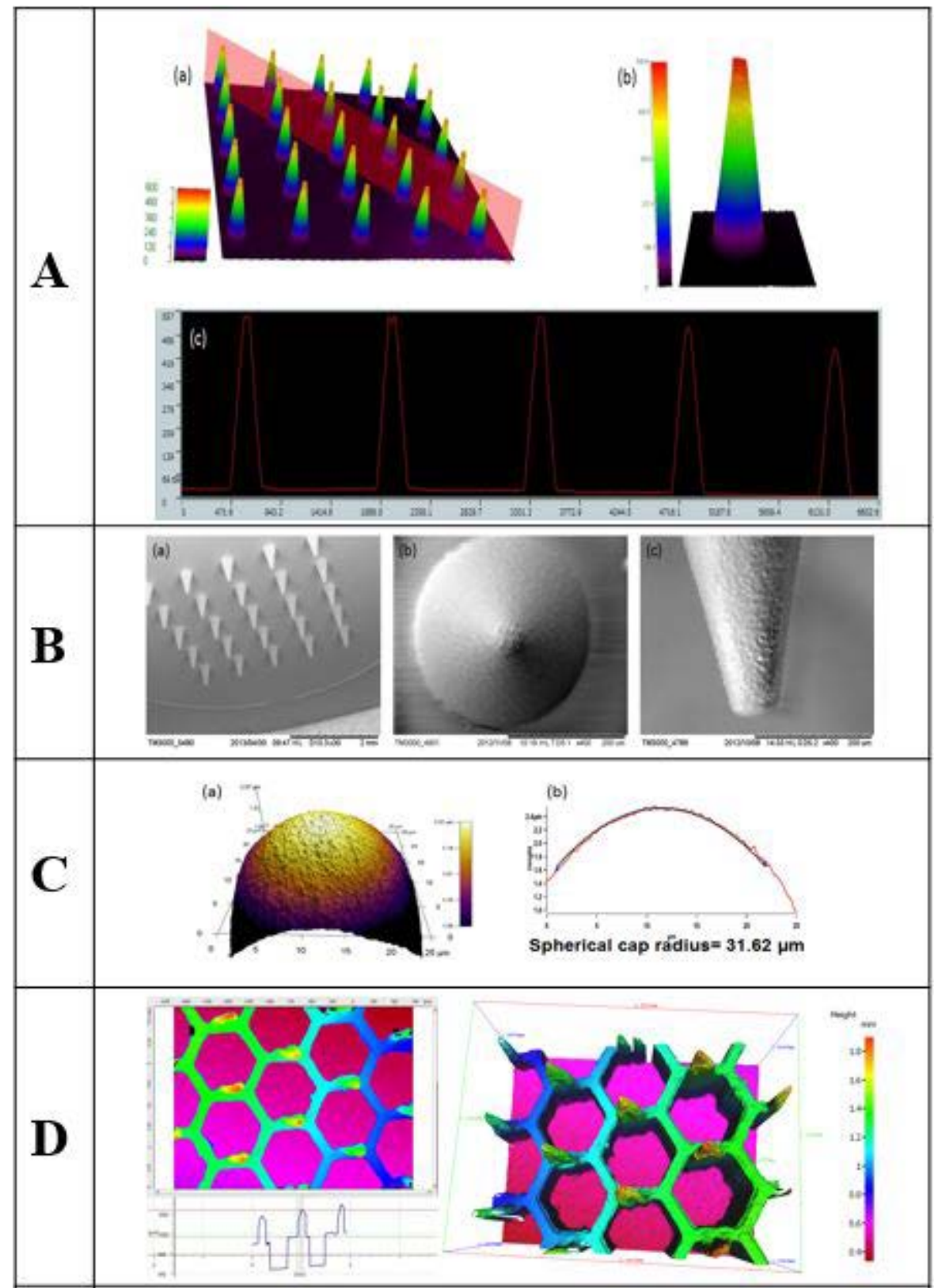

Fig 3. (A) 3D confocal image of (a) front view of the PEEK MN array (b) Single MN of PEEK (c) line measurement of the PM array; (B) SEM image of (a) MN array of PEEK (b) top view of the needle (c) Single MN of PEEK; (C) AFM image of the PEEK MN tip (b) line graph showing the spherical cap radius of the needle; (D) 3D confocal image of $0.6 \mathrm{~mm}$ ADM showing its design parameters 

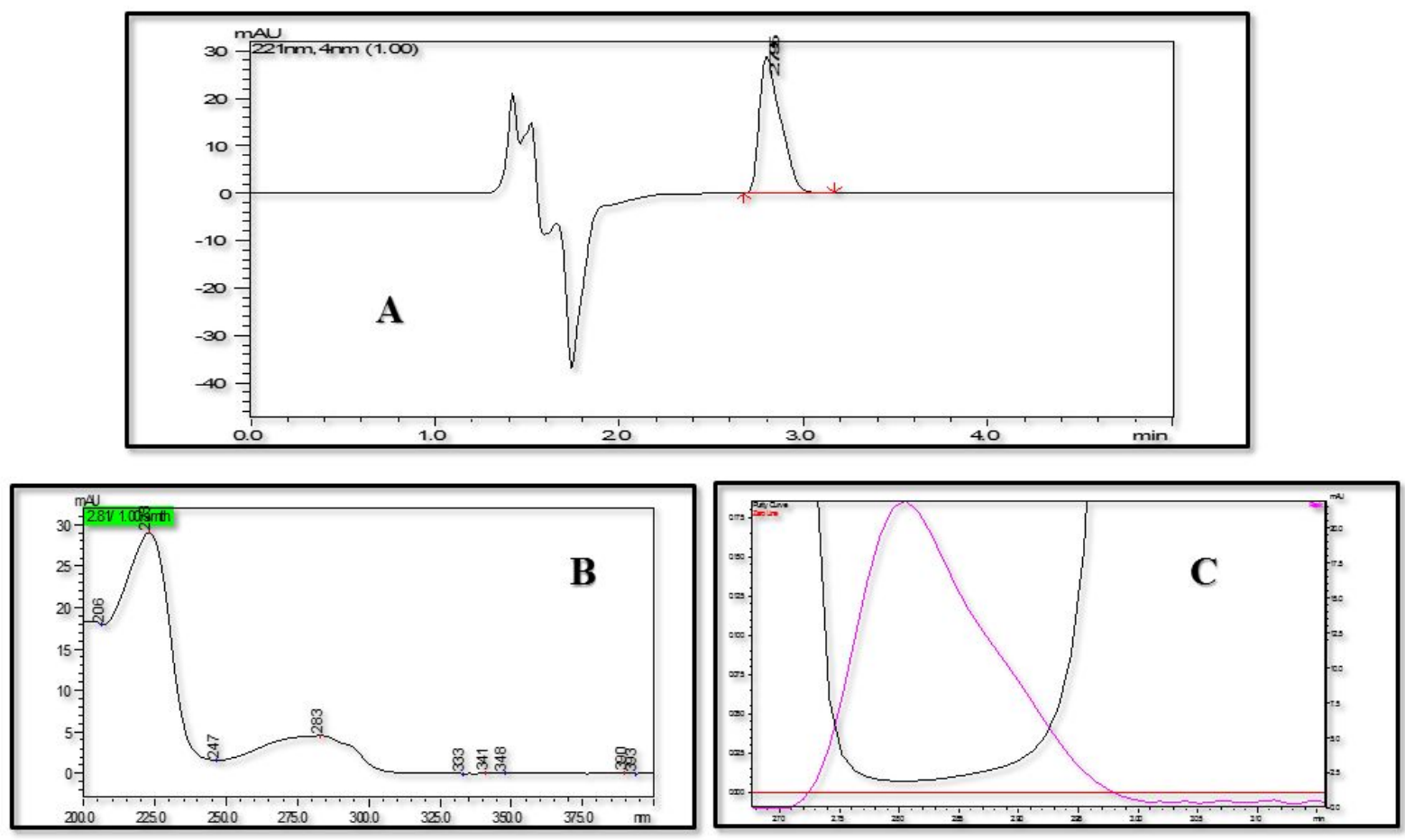

Fig 4. A - RIZ Chromatogram; B - UV Spectrum; C - Peak Purity Index 


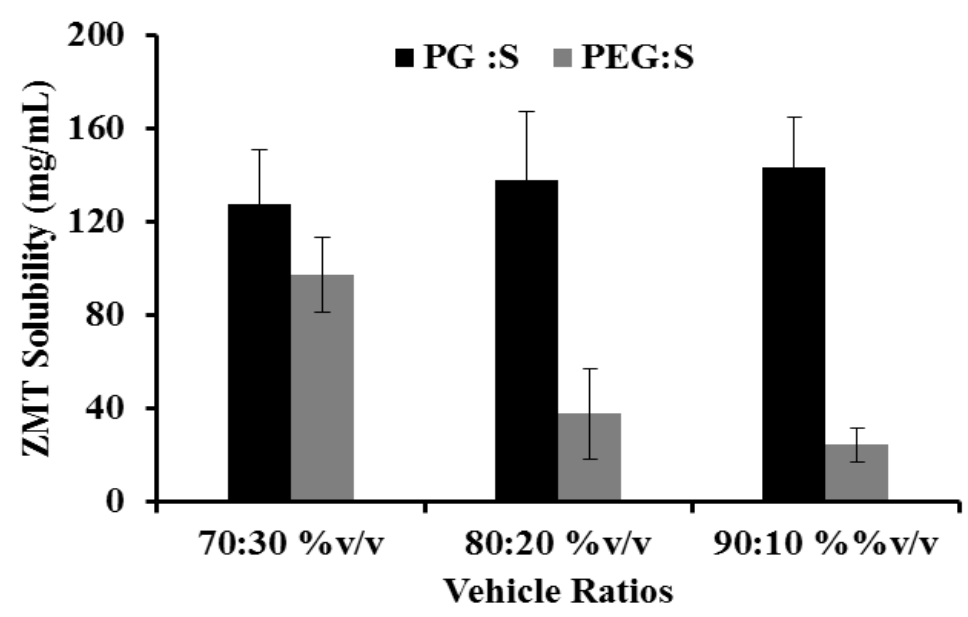

Fig 5. Solubility Data of ZMT in PG:S and PEG:S Solvent Systems 

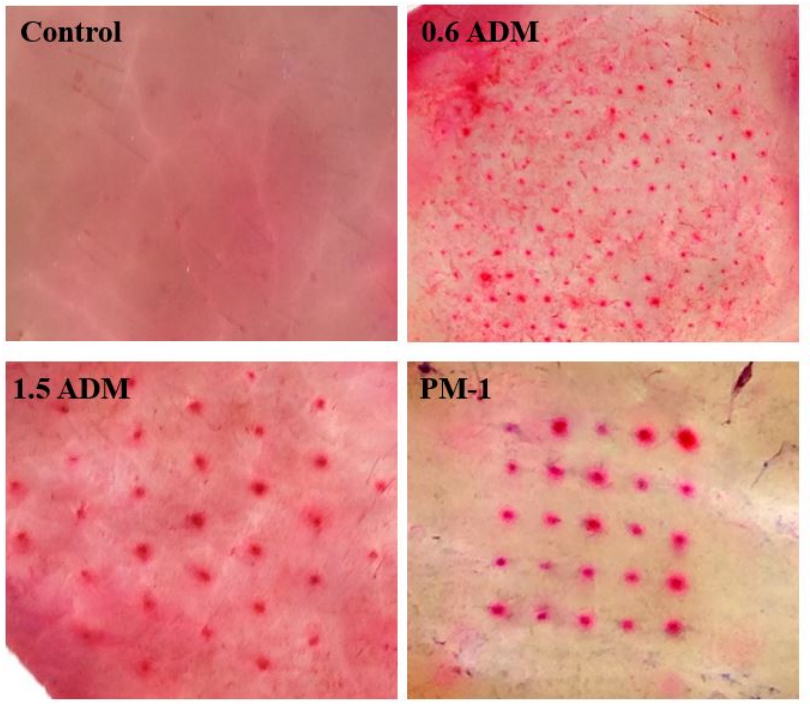

Fig 6. Surface images of stained skin without and with $\mathrm{MN}$ treatment
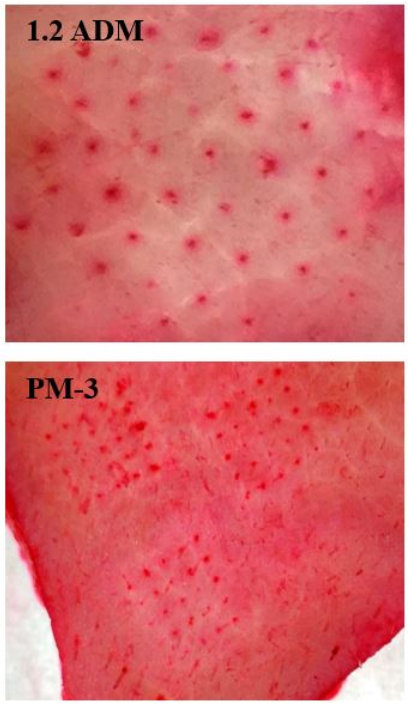


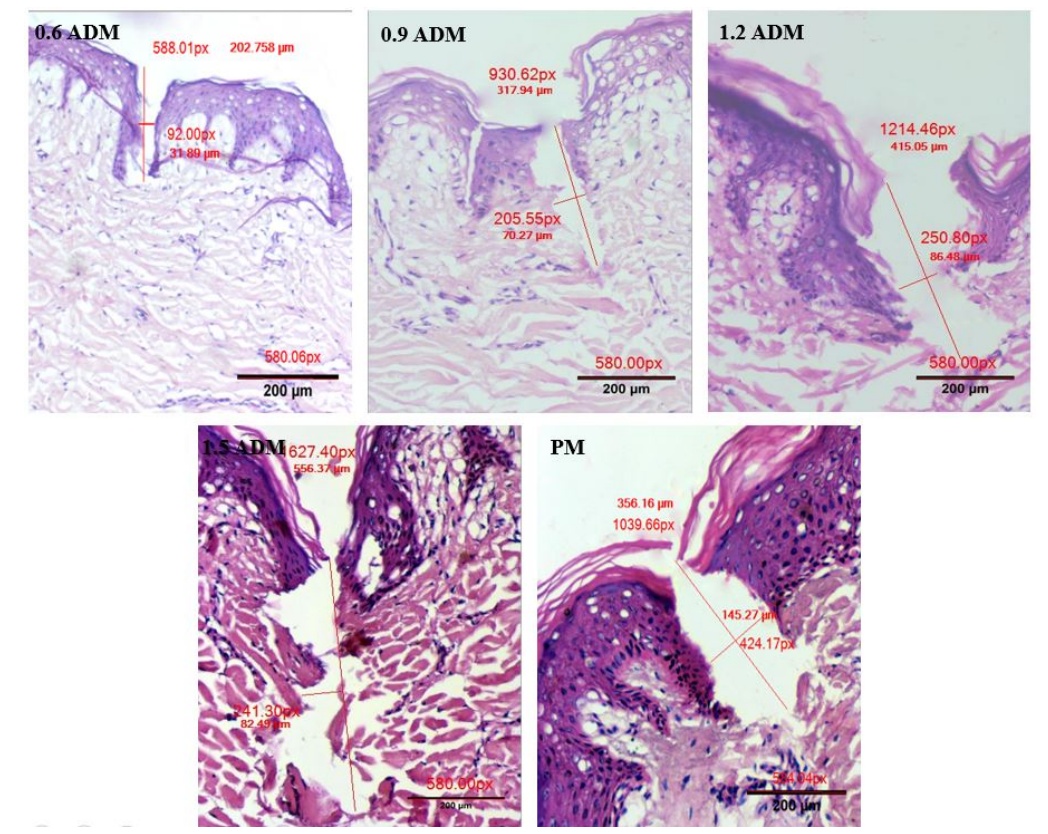

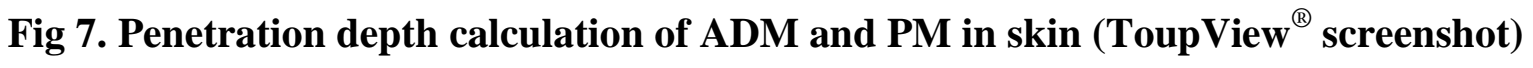




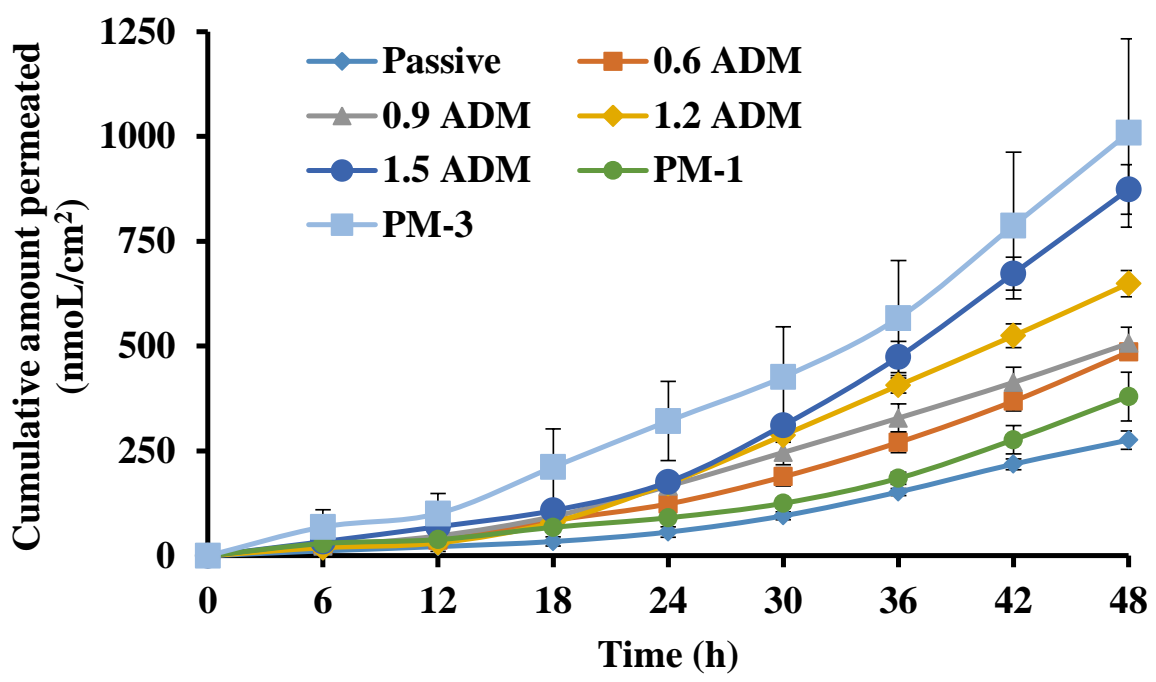

Fig 8. Comparative in vitro skin permeation profiles of ZMT from passive and MN treatments. 

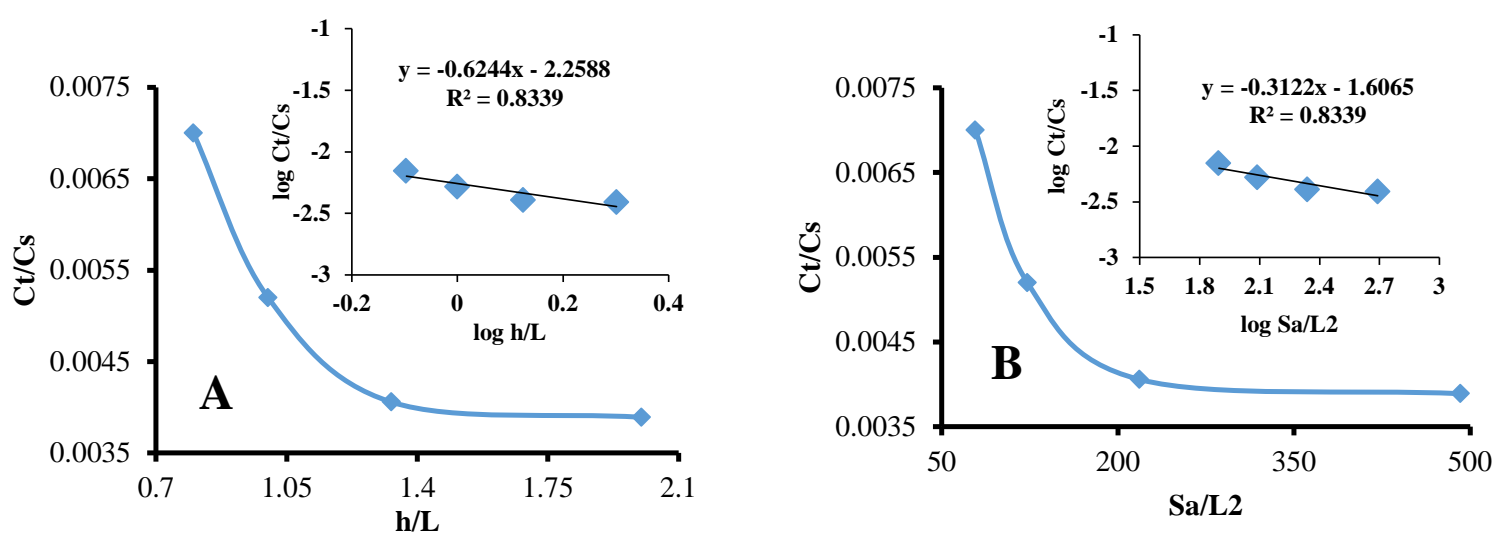

Figure 9. Scaling relationship of different dimensionless groups for permeation of RIZ(A) Effects of dimensionless length of MNs; (B) Effects of dimensionless surface area for diffusion 


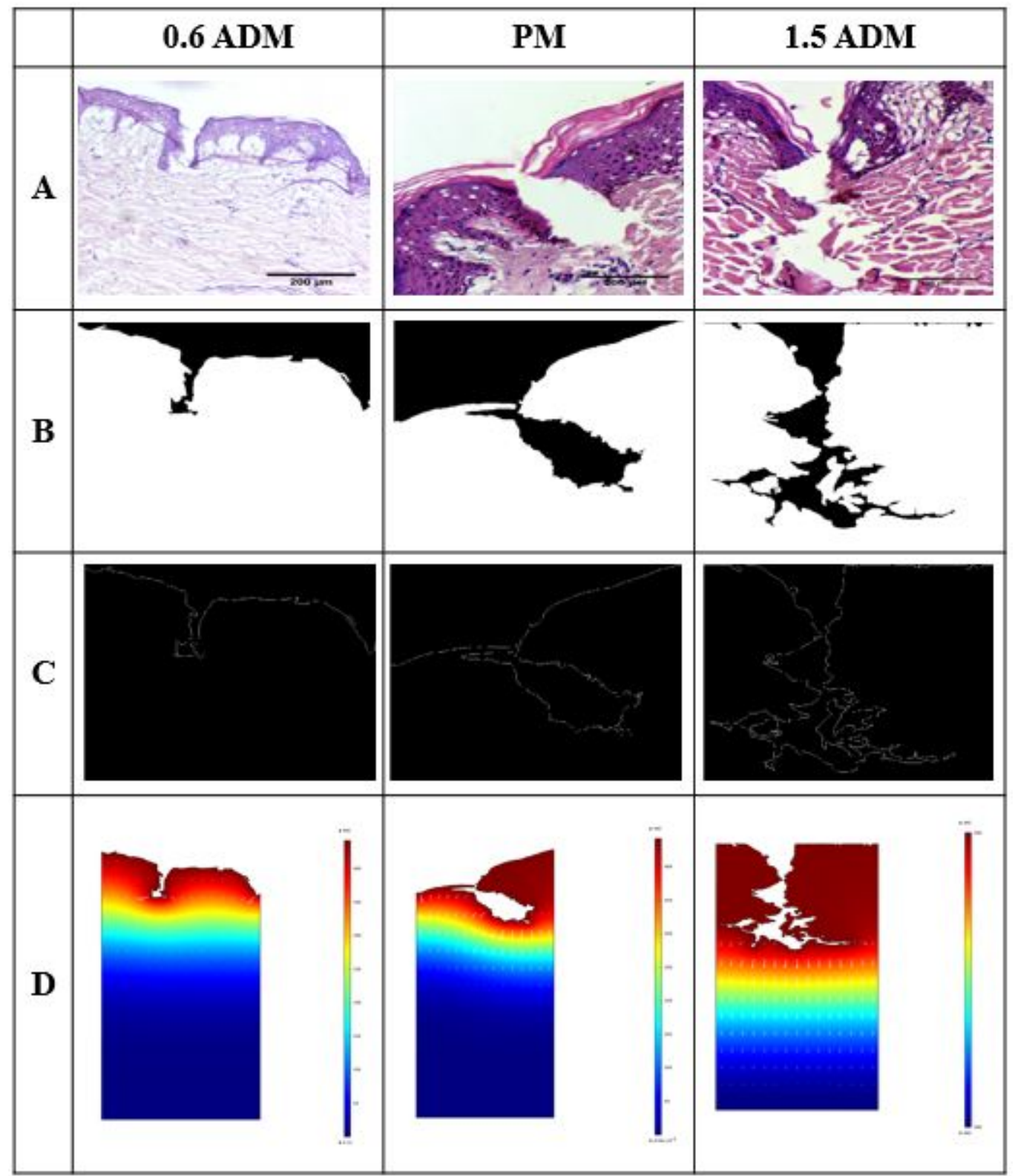

Fig 10. A - The histological section images of skin. B, C - Images treated and processed with MATLAB program. D - Images simulated in COMSOL 